\title{
Frequency Assignment for Cellular Mobile Systems Using a Memetic Algorithm
}

\author{
Tounsi Abdelkader ${ }^{1}$, Babes Malika ${ }^{2}$ \\ \#1 University Badji Mokhtar, Department of Computer Science, Laboratory of networks and system \\ BP 1223000 Annaba, Algeria \\ tounsi.abdelkader@hotmail.com \\ \#2 University Badji Mokhtar, Department of Computer Science \\ BP 1223000 Annaba, Algeria, \\ malikababes@yahoo.fr
}

\begin{abstract}
This paper presents a novel algorithm for finding a solution to the frequency assignment problem (FAP). The algorithm proposed here is a metaheuristic approach called memetic algorithm, which uses genetic algorithm based on new crossover operator combined with a modified tabu search. The performance of our algorithm is evaluated using for the results eight well-known benchmark problems, Computational results allow us to confirm the effectiveness of the proposed algorithm.
\end{abstract}

Keywords: frequency assignment problem; genetic algorithm; tabu search; memetic algorithm, metaheuristic.

\section{INTRODUCTION}

Due to the insufficiency of available bandwidth resources and the increasing demand for cellular communication services, the Frequency assignment problem becomes increasingly important.

Frequency assignment is generalization of the graph coloring problem, which belongs to the class of NP-complete problems [4]. For this category of problem, there is no known algorithm that can generate a guaranteed optimal solution in an execution time that may be expressed as a finite polynomial of the problem dimension. As this reason many heuristic methods have been proposed to deal with FAP. This large production has been analyzed and organized in several surveys [1, 2, 3]. We briefly present about each of used approaches:

- genetic algorithm based approaches.

- Constructive algorithms and local searches.

- Tabu Search.

- hybrid algorithm.

- $\quad$ approach based on ant colony paradigms.

- Neural Network Algorithm

- Simulated anneling

- Multi agent Systems

From the experiments made by most researchers on a variety of standard benchmark problems, tabu search(TS) has been one of the most effective heuristic algorithms for the FAP especially when solution time is less important than solution quality [5]. The differences in these approaches lie in the representation of the move, in the definition of neighborhood of a move, and in the way of defining a tabu move [1]. Moreover genetic algorithm (GA) provides for a wide exploration of the space to search. As these reasons the hybridization between the two last approaches is meant to accelerate the discovery of good solutions.

Memetic algorithms (MAs), which are similar to genetic algorithms, are good algorithms for combinatorial optimization problems [14]. Normally, a genetic algorithm combined with local search methods is called a memetic algorithm (MA) [14]. MAs have received various names throughout the literature and scientists not always agree what is and what is not an MA due to the large variety of implementations available. Some of the alternative names used for this search framework are hybrid GAs, Baldwinian EAs, Lamarckian EAs, genetic local search algorithms, and other names are not unheard of. Moscato [13] coined the name Memetic Algorithm to cover a wide range of techniques where evolutionary-based search is augmented by the addition of one or more phases of local search.

In this paper, we propose a memetic approach for solving the frequency assignment problem. We consider a general cellular radio network subjected to all three kinds of constraints [8] :

1. the cochannel constraint (CCC): the same frequency cannot be assigned to certain pairs of radio cells simultaneously;

2. the adjacent channel constraint(ACC): frequencies adjacent in the frequency domain cannot be assigned to adjacent radio cells simultaneously;

3. the co-site constraint (CSC): any pair of frequencies assigned to a radio cell must have certain distance in the frequency domain.

The rest of the paper is organized as follows. In Section 2, we formulate the frequency assignment problem. In Section 3, we present the proposed algorithm. In Section 4, experimental results are presented. Future work and perspectives are discussed in the last section. 


\section{PROBLEM DESCRIPTION}

The problem of frequency assignment is to provide wireless communication frequencies from limited spectral resources while keeping to a minimum interference suffered by those wishing to communicate in a given radio communication network. According to [2] the here considered version of FAP is classified as a MS-FAP (minimum Span frequency assignment problem). Radio channels are represented by the positive integers. Let $\mathrm{M}=\{1,2, \ldots, \mathrm{m}\}$ be a set of available channels where $m$ is the Number of available channels in the mobile network. The basic model of the channel assignment problem can be represented as follows:

1. $N$ : The number of cells in the mobile network.

2. $d_{i}$ : represents the number of frequencies that must be assigned to cell $i(1 \leq i \leq N)$

3. $C$ : Compatibility matrix, $C=\left(c_{i j}\right)_{N x N}$ represents the Minimal channel separation between channels in cells $i$ and $j, 1 \leq i$, $j \leq N$.

4. $\quad$ Call $_{i k}$ : Cell $i$ with call $\mathrm{k}$ where $1 \leq \mathrm{i} \leq \mathrm{N}, 1 \leq \mathrm{k} \leq \mathrm{d}_{\mathrm{i}}$.

5. $f_{i k}:$ A radio channel is assigned to $\mathrm{Call}_{\mathrm{ik}}$, where $f_{i k}$ belongs to the set of radio channel $\mathrm{F}$.

6. Frequency separation constraint :

$\left|f_{i k}-f_{j m}\right| \geq \mathrm{c}_{\mathrm{ij}}$, for all $\mathrm{i}, \mathrm{j}, \mathrm{k}, \mathrm{m}(\mathrm{i} \neq \mathrm{j}, \mathrm{k} \neq \mathrm{m}), \mathrm{c}_{\mathrm{ij}}$ is defined in Compatibility Matrix, C. If $(\mathrm{i}=\mathrm{j})$, it become co-site constraint.

7. TotalAssignCh: the total number of required channels The total of radio channel to be assigned in the system can be shown as:

$$
\text { TotalAssignCh }=\sum_{1}^{\mathrm{n}} \mathrm{d}(\mathrm{i})
$$

Therefore, the objective is to find an assignment that minimizes the total number of violations in an assignment, Subject to:

$$
\min \sum_{\mathrm{i}=1}^{\mathrm{n}} \sum_{\mathrm{k}=1}^{\mathrm{m}} \sum_{\mathrm{j}=1}^{\mathrm{n}} \sum_{\mathrm{l}=1}^{\mathrm{m}} \mathrm{f}_{\mathrm{ik}} \operatorname{viol}(\mathrm{i}, \mathrm{j}, \mathrm{k}, \mathrm{l}) \mathrm{f}_{\mathrm{j} 1}
$$

Where: $\quad \operatorname{viol}(\mathrm{i}, \mathrm{j}, \mathrm{k}, \mathrm{l})=\left\{\begin{array}{cc}0 & \text { if }|\mathrm{k}-\mathrm{l}| \geq C_{i j} \\ 1 & \text { otherwise }\end{array}\right.$ and

$$
\begin{gathered}
\mathrm{f}_{\mathrm{ik}}= \begin{cases}0 & \text { if channel } \mathrm{k} \text { is not assigned to cell } \mathrm{i} \\
1 & \text { otherwise }\end{cases} \\
\text { for } 1 \leq k, l \leq m \text { and } 1 \leq \mathrm{i}, \mathrm{j} \leq N .
\end{gathered}
$$

\section{MEMETIC ALGORITHM}

In this paper, a Memetic Algorithm (MA) is proposed for FAP. The mechanisms and operators of the MA are given below.

\section{A. Representation and selection mechanisms}

Encoding the chromosome in FAP is relatively simple, We represent a solution by a binary matrix $N \times m . N$ is the number of cells and $\mathrm{m}$ is the number of channels. If a gene $f_{i k}=1$, then the $k^{\text {th }}$ channel is assigned to the $i^{\text {th }}$ cell. Chromosomes are randomly selected from the population for crossover and mutation.

\section{B. Population initialization}

In a traditional GA, each chromosome in the initial population is generated randomly. However, many experimental results have shown that the GA does not always result in good solutions due to the random method.

the co-site constraint (CSC) may cause more interference in the real-world situation[6]. So firstly to make sure the absence of interference between two channels assigned to the same cell, we have generate the initial population with new method called (PI) inspired from[7]. Figure 1 shows the difference between the two methods: -Cell: $\mathrm{i}$

- $\mathrm{j}$ : is random number between 1 and $\left.\mathrm{m}-\left(\left(\mathrm{d}_{\mathrm{i}}-1\right) \times \mathrm{C}_{\mathrm{ii}}+1\right)\right)$



(a)

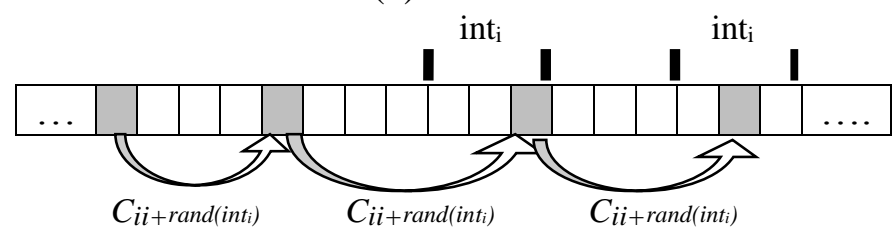

(b)

Fig.1: Frequencies assigned to cell 'i'

(a) Cheng method [7] (b) our method(PI)

The int $_{i}$ is defined by

$$
\operatorname{Int}_{\mathrm{i}}=\frac{\mathrm{m}-\left(\left(\mathrm{d}_{\mathrm{i}}-1\right) \times \mathrm{C}_{\mathrm{ii}}+1\right)}{\mathrm{d}_{\mathrm{i}}}
$$

Where:

$\mathrm{C}_{\mathrm{ii}}$ represents the Minimal channel separation between channels in cell $i$;

$\mathrm{Int}_{\mathrm{i}}+\mathrm{C}_{\mathrm{ii}}$ is the maximum channel separation between channel in cell i ;

Int $_{i}$ represents the interval of possible assignment to cell "i" without breaking the CSC constraint of all assignment in the Cell i.

The cells with a high channel demand have more difficulty obtaining an assignment that satisfies the CSC constraints. So we have generated $p_{i}$ to deal with this problem. Where $p_{i}$ is defined as:

$$
\mathrm{p}_{\mathrm{i}}=\frac{\left(\mathrm{d}_{\mathrm{i}}-1\right) \times \mathrm{C}_{\mathrm{ii}}+1}{\mathrm{~m}}
$$


Cells with a high value of $p_{i}$ means that these cells must use the minimum separation distance $\left(\mathrm{C}_{\mathrm{ii}}\right)$ between channels. For the others with low value of $p_{i}$ means that these cells use the separation distance $\mathrm{C}_{\mathrm{ii}}+$ random $\left(0\right.$, int $\left._{\mathrm{i}}\right)$.Figure 2 is the pseudo code of PI. Where sol $\mathrm{i}_{\mathrm{i}}$ is the assignment of cell $\mathrm{i}$.

\section{Proposed crossover operator}

Chromosomes in the population have good genes resulting from the population initialization, It is clear that performance can be improved by transferring these good genes to the next generation.

An overview of the crossover operator is shown by figure 3 . Two parents such as parent 1 and parent 2 are considered for the crossover process. We randomly generate a binary number mask then, we calculate $P_{i}$ for each cell in the tow parents where $\mathrm{P}_{\mathrm{i}}$ is defined in (4). The value of $\mathrm{Pi}$ can exclusively reduce the searching space and consequently the convergence time is shortened. $\mathrm{P}_{\mathrm{i}}$ has two roles; the first one is keeping CSC constraints for some cells verified, the other one is for the cells that do not have a high probability for improvement - optimum solution obtained -will be leaved uncrossed.

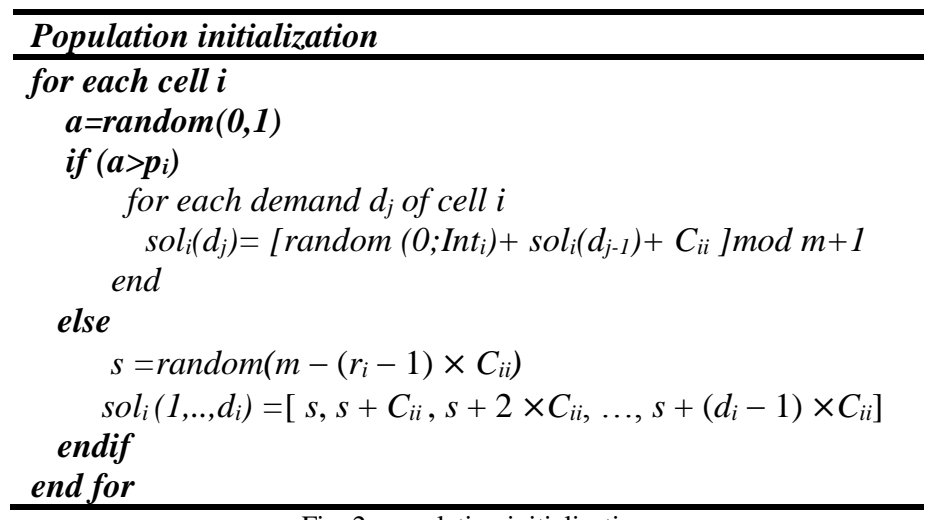

$\mathrm{C}=$ Random $(0,1)$

If $\left(\mathrm{C}<\mathrm{p}_{\mathrm{i}}\right)$

The row pairs leave uncrossed

Else

The row pairs will be crossed

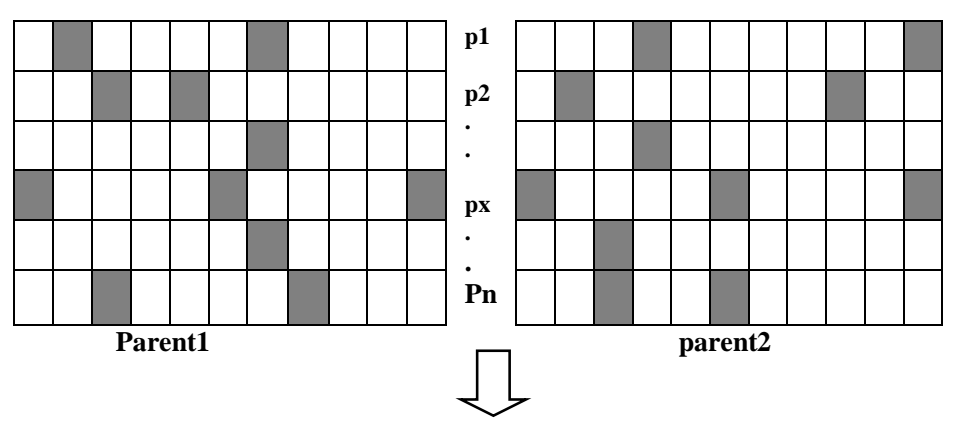

\begin{tabular}{|l|l|l|l|l|l|l|l|l|l|l|}
\hline $\mathbf{1}$ & $\mathbf{0}$ & $\mathbf{0}$ & $\mathbf{0}$ & $\mathbf{1}$ & $\mathbf{1}$ & $\mathbf{1}$ & $\mathbf{0}$ & $\mathbf{1}$ & $\mathbf{0}$ & $\mathbf{1}$ \\
\hline $\mathbf{0}$ & $\mathbf{0}$ & $\mathbf{0}$ & $\mathbf{0}$ & $\mathbf{0}$ & $\mathbf{0}$ & $\mathbf{0}$ & $\mathbf{0}$ & $\mathbf{0}$ & $\mathbf{0}$ & $\mathbf{0}$ \\
\hline $\mathbf{1}$ & $\mathbf{0}$ & $\mathbf{1}$ & $\mathbf{1}$ & $\mathbf{0}$ & $\mathbf{1}$ & $\mathbf{1}$ & $\mathbf{0}$ & $\mathbf{1}$ & $\mathbf{0}$ & $\mathbf{0}$ \\
\hline $\mathbf{1}$ & $\mathbf{1}$ & $\mathbf{0}$ & $\mathbf{0}$ & $\mathbf{1}$ & $\mathbf{1}$ & $\mathbf{0}$ & $\mathbf{1}$ & $\mathbf{0}$ & $\mathbf{0}$ & $\mathbf{0} \mathbf{P})$ \\
\hline
\end{tabular}

Binary mask

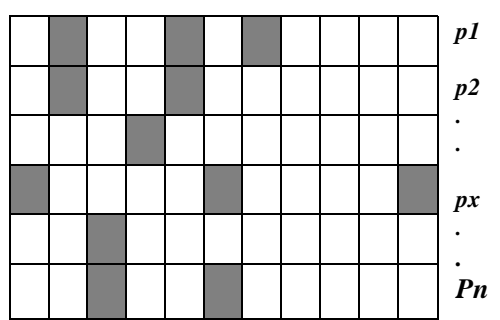

First kids

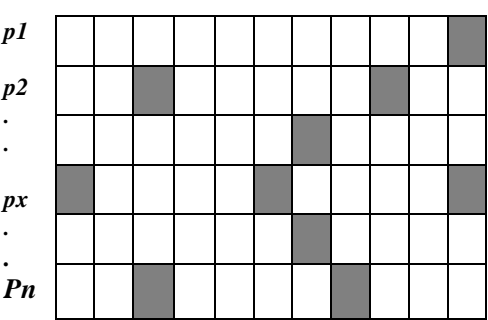

Second kids
Fig. 3: Proposed crossover operator

\section{Mutation operator}

Our mutation operator called "mutation adapter" controls the total number of " 1 " in row $i$. we make sure that not to exceed $d_{i}$ of the demand vector in any case. We do this by adding or removing a " 1 " randomly from the specified row. The mechanism of mutation operator is shown in figure 4 .
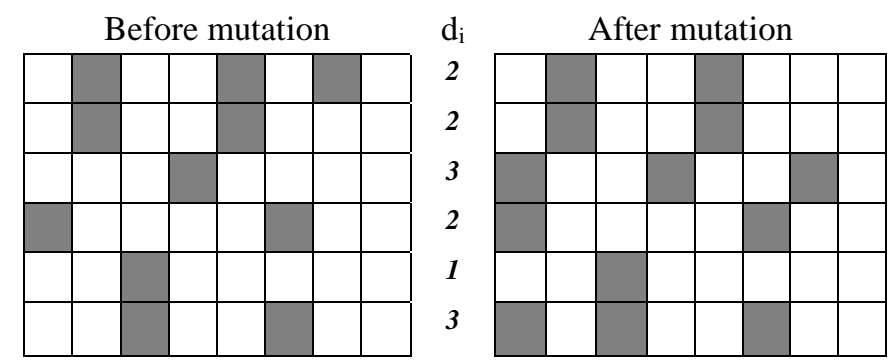

Fig. 4: mutation adapter

\section{E. MA description}

Figure 5 illustrates the MA framework followed in the implementation of the proposed algorithm. The first step of the algorithm is to create an initial population with PI and evaluate it .After that, for each generation, a pair of "parent" solutions is selected for the above methods of crossover and mutation. Two solutions are created which typically shares many of the characteristics of its "parents. After that the Tabu search and the module of restricted frequency are performed on these solutions. The mechanisms and operators of these components are given in section $\mathrm{F}$ and $\mathrm{G}$.

\section{Algorithm $M A$}




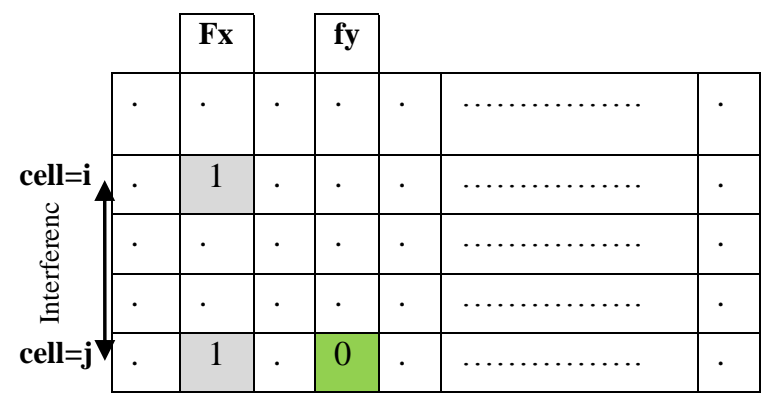

\begin{tabular}{|l|l|l|l|l|l|l|}
\hline$\cdot$ & $\cdot$ & $\cdot$ & $\cdot$ & $\cdot$ & $\ldots \ldots \ldots \ldots \ldots \ldots$ & $\cdot$ \\
\hline$\cdot$ & 1 & $\cdot$ & & $\cdot$ & $\ldots \ldots \ldots \ldots \ldots \ldots$ & $\cdot$ \\
\hline$\cdot$ & $\cdot$ & $\cdot$ & $\cdot$ & $\cdot$ & $\ldots \ldots \ldots \ldots \ldots \ldots \ldots \ldots \ldots \ldots$ & $\cdot$ \\
\hline$\cdot$ & $\cdot$ & $\cdot$ & $\cdot$ & $\cdot$ & $\ldots \ldots \ldots \ldots \ldots \ldots \ldots \ldots$ & $\cdot$ \\
\hline
\end{tabular}

\section{Begin}

Initialize population $P$ with $P I$;

Evaluate initial population,

Repeat

-Generate-Binary-Masks

-Select two parents randomly

-use the proposed crossover. Then get two offspring

- mutation adapter(ofspring 1, ofspring2)

- If fitness (ofspring2) better than fitness (parent)

then Ofspring will be accepted.

- tabu Search of s ing

- calculate the unrestricted freque los for each cell

which have interference in ofspringl

- replace the restricted frequencies with

unrestricted frequencies

-add ofspringl to population

Until Termination criteria are satisfied;

End;

Fig. 5: MA framework

\section{F. Tabu search}

After several generations, chromosomes in the population are similar enough to each other such that only local optimization may be possible. Tabu search is one of the mechanisms to avoid minimum local. Opposite to randomizing approaches such as Simulated Annealing (SA) where randomness is widely used, TS is based on the principle that intelligent search must embrace more efficient and systematic forms of direction such as memorizing and learning [15].the hybridization between GA and TS is more successful in our case.

\section{1) Solution representation}

The representation of a frequency assignment $\mathrm{S}$ is obtained by using a matrix $\mathrm{N} \times \mathrm{m}$. where if a gene $\mathrm{f}_{\mathrm{ik}}=1$, then the $\mathrm{k}^{\text {th }}$ channel is assigned to the $\mathrm{i}^{\text {th }}$ cell.

\section{2) Neighborhood}

The space to search or set of moves is defined in figure 6. Our basic move is then moving a 1 to a 0 entry of the same row. Thus changing the assigned channel of one cell, maintaining the number of assigned channels unchanged and keeping the result of crossover operator (co-site interference) .

\section{3) Tabu list}

Some neighbors composed of previously encountered solutions will not be considered for the next $\mathrm{k}$ iterations $(\mathrm{k}$, called tabu length). These neighbors are consisted in tabu list, which is one of the main mechanisms of tabu search. Tabu list is also the most important feature distinguished with other search algorithms. Thus, TS can be described as a form of neighborhood search with a set of critical and complementary components.

As shown in Figure 6, the element (j, fx) prevents the algorithm from re-visiting previously seen solutions. The element $(j$, fy) prevents the other individuals from re-exploring the same search area. tabulist $=(\mathbf{j}, \mathbf{f x}) ;(\mathbf{j}, \mathbf{f y})$

Fig. 6: Tabu search moves

\section{G. Restricted frequencies}

In order to improve the quality of the solution obtained, we apply the following steps to locate the free frequencies (if any exist) and resolve the maximum of conflict between cells. This 
module is applied to solution obtained by tabu search that do not satisfy all the constraints of the problem.

1. determine the couples of cells which have interference between them ;

2. from the list of couples " $\mathrm{S}$ ", we create list of candidate $\mathrm{L}$. where $\mathrm{L}$ is defined by:

$\mathrm{L}=\left\{\mathrm{x}, \mathrm{p}_{\mathrm{x}}<\mathrm{p}_{\mathrm{y}} \forall(\mathrm{x}, \mathrm{y}) \in \mathrm{S}\right\} \mathrm{p}_{\mathrm{x}}, \mathrm{p}_{\mathrm{y}}$ defined in (4)

3. delete all frequencies assigned to $\mathrm{L}$

4. Assigned $=\{\}$

5. calculate" free frequencies" it means the authorization of assigning frequencies in cell $\mathrm{x}, \forall \mathrm{x} \in(\mathrm{L}-$ Assigned $)$ (see figure 7) if (L-Assigned $)=\{\}$ go to 7;

6. if ( free frequencies) then $\mathrm{x} \in$ (L- Assigned) Assign the first free frequency to " $\mathrm{x}$ ", Assigned $=\{x\}$; else

Assign a frequency randomly to $\mathrm{x}$;

Assigned $=\{\mathrm{x}\}$; endif ; go to 5.

7. End

This approach shares some similarities in the calculation of the free frequency in Frequency Exhaustive Strategy [16].

\section{H. Termination criteria}

In traditional GA, either computation time or the number of generations or solution improved is selected as termination criterion. In this paper, all of them are considered. If a solution cannot be improved any more in consecutive generations, the algorithm terminates. However, it is very time-consuming for problems, MA terminates when any of the three conditions is true.



Assign these free frequencies to cell $x$ make no interference between $\mathrm{x}$ and cells $\mathrm{i}$ and $\mathrm{j}$

Fig. 7: Free frequencies

\section{EXPERIMENTAL RESULTS}

In these experiments we used the Philadelphia problem instances [12]. These instances are widely known within problem FAP, and they are characterized by a number of hexagonal cells (habitually 21), that represent a cellular phone network of the Philadelphia city (see Figure 8).

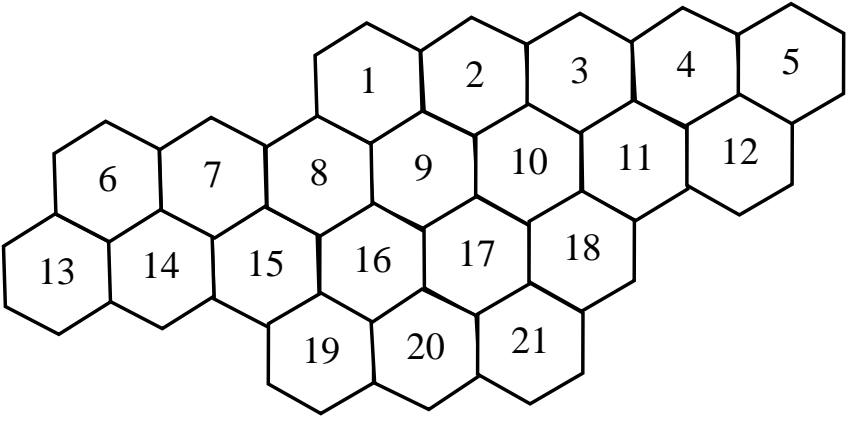

Fig. 8: Cellular geometry of test problems

The main differences among the instances correspond with the use of different interference matrices and demand vectors (see table 1). In these instances, the number of antennas (cells) varies from 4 to 25 , and the number of frequencies finally used varies between 11 and 533. Figure 11 shows the different interference matrices $(C)$ and the demand vectors (D) used.

Table I: Problem Specifications

\begin{tabular}{c|c|c|c|c}
\hline Instance & $\begin{array}{c}\text { \# of } \\
\text { Cell }\end{array}$ & $\begin{array}{c}\text { Lower } \\
\text { Bound } \\
(\mathrm{lb})\end{array}$ & $\begin{array}{l}\text { Compatibility } \\
\text { matrix }(C)\end{array}$ & $\begin{array}{l}\text { Demand } \\
\text { vector } \\
(\mathrm{D})\end{array}$ \\
\hline P1 & 4 & 11 & $\mathrm{C}_{1}$ & $\mathrm{D}_{1}$ \\
P2 & 25 & 73 & $\mathrm{C}_{2}$ & $\mathrm{D}_{2}$ \\
P3 & 21 & 381 & $\mathrm{C}_{3}$ & $\mathrm{D}_{3}$ \\
P4 & 21 & 533 & $\mathrm{C}_{4}$ & $\mathrm{D}_{3}$ \\
P5 & 21 & 533 & $\mathrm{C}_{5}$ & $\mathrm{D}_{3}$ \\
P6 & 21 & 221 & $\mathrm{C}_{3}$ & $\mathrm{D}_{4}$ \\
P7 & 21 & 309 & $\mathrm{C}_{4}$ & $\mathrm{D}_{4}$ \\
P8 & 21 & 309 & $\mathrm{C}_{5}$ & $\mathrm{D}_{4}$ \\
& & & & \\
\hline
\end{tabular}

To investigate the convergence frequency, Table II summarizes the simulation results, and shows the convergence to the optimum solution (the convergence frequency).

Table II: convergence rate

\begin{tabular}{l|l|l|c|c|c|l}
\hline Inst & \multicolumn{6}{|l|}{ Approach and rate of convergence } \\
\cline { 2 - 7 } & $\begin{array}{l}\text { Lower } \\
\text { Bound }\end{array}$ & MA & $\begin{array}{c}{[9]} \\
\text { MGA }\end{array}$ & $\begin{array}{c}{[10]} \\
\text { NN }\end{array}$ & $\begin{array}{l}{[11]} \\
\text { DGuGA }\end{array}$ & $\begin{array}{l}{[7]} \\
\text { CSCP }\end{array}$ \\
\hline P1 & 11 & $100 \%$ & $100 \%$ & - & $100 \%$ & $100 \%$ \\
\hline P2 & 73 & $100 \%$ & $100 \%$ & $62 \%$ & $98 \%$ & $100 \%$ \\
\hline P3 & 381 & $100 \%$ & - & $99 \%$ & - & $100 \%$ \\
\hline P4 & 533 & $100 \%$ & - & $100 \%$ & - & $100 \%$ \\
\hline P5 & 533 & $100 \%$ & - & $98 \%$ & - & $100 \%$ \\
\hline P6 & 221 & $100 \%$ & $92 \%$ & $97 \%$ & $89 \%$ & $100 \%$ \\
\hline P7 & 309 & $100 \%$ & - & $99 \%$ & - & $100 \%$ \\
\hline P8 & 309 & $100 \%$ & $80 \%$ & $52 \%$ & - & $100 \%$ \\
\hline
\end{tabular}


Figure 9 shows the required generation to converge to the solution in two problems. In problem \#8, our MA found the solution within 22 generation, in problem \#2, our MA converge in the 36 th generation.

Table 2 allows us to confirm the effectiveness of the proposed algorithm. The simulation results show that our algorithm achieved $100 \%$ convergence to solutions for all eight benchmarking problems. The results show that MA outperform the convergence results of the neural network (NN) [10], genetic algorithm (MGA) (DGuGA) [9,11]. The MA shows the same convergence results of [7]. Our algorithm identifies higher-quality solutions than other methods and it is easy to implement. For cellular systems with high number of cells, this algorithm can be efficiently applied to find the exact solution in an acceptable time of computation. Also since it is an implicitly parallel technique, it can be implemented very effectively on powerful parallel computers to solve exceptionally demanding large-scale problems.

Figure 10 shows a two-dimensional stem plot. It displays the frequency assigned to each cell for the problem \#8, where the $\mathrm{x}$-axis represent the cells, and the $\mathrm{y}$-axis represent the frequency assigned . The full assignment of channels for a sample of the 8 benchmark problems considered in our simulations is presented in the Appendix. The results and analysis presented above indicate that our MA is certainly an effective Memetic algorithm for solving the frequency assignment problem.



(a)

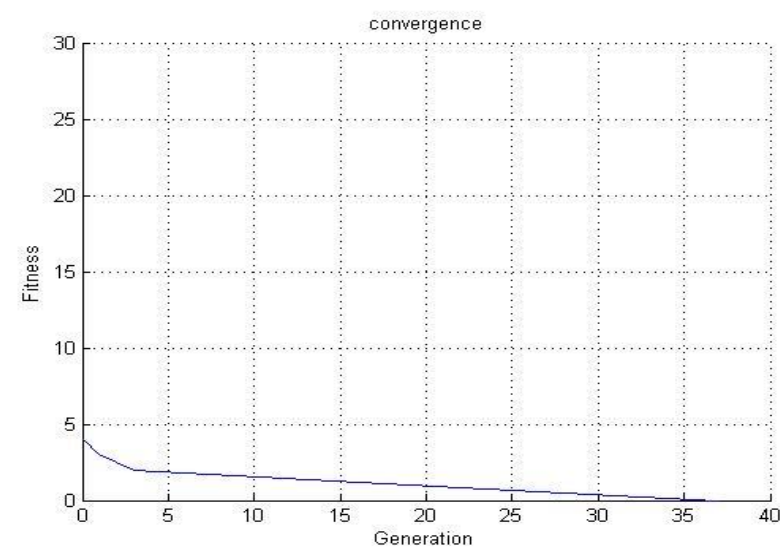

(b)

Fig. 9: (a)instance $\mathrm{P} 8$ convergence (b) instance $\mathrm{P} 2$ convergence

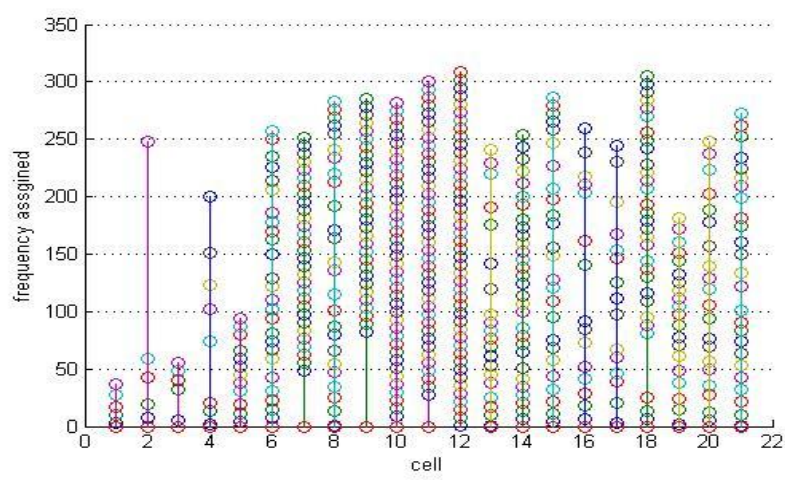

Fig. 10: two-dimensional stem plot

\section{CONCLUSION}

We have presented a memetic algorithm for solving the frequency assignment problem in cellular radio networks. Due the importance of CSC constraints, we have considered this in the population initialization. Problems specific information has been used into the mechanism of crossover and tabu search as to effectively bias the search process towards promising regions of the search space. After experiments with real data, optimization was achieved and the algorithm has been efficient and convergent. Our approach can find better or equivalent solutions compared with existing optimization methods.

Our work presents a good perspective of managing the radio spectrum that can be intended towards a parallel implementation for implementing a pertinent strategy and effective management of resources in the future works.

\section{Appendix:}

This appendix shows the full assignments for benchmark instances No. 2, 5, and 6(see table 3-5)

Table III: Channel assignment for benchmark problem 2 
ICIT 2015 The 7th International Conference on Information Technology

doi:10.15849/icit.2015.0040 (C) ICIT 2015 (http://icit.zuj.edu.jo/ICIT15)

\begin{tabular}{c|l}
\hline Cells & \multicolumn{1}{|c}{ Frequency } \\
\hline 1 & \multicolumn{1}{|c}{} \\
2 & $\mathbf{3 1 , 3 3 , 3 5 , 3 7 , 3 9 , 4 1 , 6 7 , 6 9 , 7 1 , 7 3}$ \\
3 & $\mathbf{6 , 8 , 1 0 , 1 2 , 1 4 , 1 6 , 1 8 , 2 0 , 2 2 , 2 4 , 2 6}$ \\
4 & $\mathbf{2 8 , 3 2 , 3 4 , 3 6 , 3 8 , 4 0 , 4 2 , 5 1 , 7 0}$ \\
5 & $\mathbf{3 0 , 4 4 , 4 6 , 4 8 , 5 0 , 5 2 , 5 4 , 5 6 , 7 2}$ \\
6 & $\mathbf{1 , 3 , 5 , 6 7}$ \\
7 & $\mathbf{5 3 , 5 5 , 5 7 , 6 1 , 6 4}$ \\
8 & $\mathbf{3 0 , 4 4 , 4 6 , 4 8 , 5 0 , 5 2 , 5 4}$ \\
9 & $\mathbf{1 0 , 1 3 , 1 5 , 1 7}$ \\
10 & $\mathbf{2 , 4 , 4 3 , 4 7 , 5 8 , 6 0 , 6 2 , 6 6}$ \\
11 & $\mathbf{1 4 , 1 6 , 1 8 , 2 0 , 2 2 , 2 4 , 3 1 , 3 5}$ \\
12 & $\mathbf{1 , 3 , 5 , 7 , 9 , 4 5 , 5 9 , 6 3 , 6 5}$ \\
13 & $\mathbf{1 1 , 1 3 , 1 5 , 1 7 , 1 9 , 2 1 , 2 3 , 2 5 , 2 7 , 2 9}$ \\
14 & $\mathbf{4 9 , 5 3 , 5 5 , 5 7 , 6 1 , 6 4 , 6 8}$ \\
15 & $\mathbf{2 8 , 3 6 , 3 8 , 5 1 , 5 9 , 6 3 , 6 5}$ \\
16 & $\mathbf{6 , 8 , 2 6 , 3 2 , 3 7 , 4 0}$ \\
17 & $\mathbf{2 , 1 0 , 1 4 , 1 6}$ \\
18 & $\mathbf{3 , 5 , 7 , 9 , 3 3}$ \\
19 & $\mathbf{1 , 1 2 , 3 4 , 3 9 , 4 1}$ \\
20 & $\mathbf{4 4 , 4 6 , 4 8 , 5 0 , 5 2 , 5 4 , 5 6}$ \\
21 & $\mathbf{2 , 4 , 9 , 3 2 , 3 7 , 4 3}$ \\
22 & $\mathbf{3 , 5 , 1 1 , 4 2}$ \\
23 & $\mathbf{7 , 1 0 , 1 3 , 2 3 , 3 6}$ \\
24 & $\mathbf{5 3 , 5 5 , 5 7 , 5 9 , 6 1 , 6 3 , 6 5}$ \\
25 & $\mathbf{1 , 8 , 2 1 , 3 3 , 4 4}$ \\
\hline
\end{tabular}

Table IV: Channel assignment for benchmark problem 5

\begin{tabular}{|c|c|}
\hline Cells & Frequency \\
\hline 1 & $6,11,21,27,95$ \\
\hline 2 & $4,10,20,30,108$ \\
\hline 3 & $1,8,16,25,31$ \\
\hline 4 & $2,7,12,18,23,35,40,45$ \\
\hline 5 & $3,9,14,20,28,33,38,43,48,55,60,65$ \\
\hline 6 & $\begin{array}{l}1,7,12,18,24,33,40,45,50,57,66,75,81,86,91,100,106,111,116,122,137,145,152 \\
191,200\end{array}$ \\
\hline 7 & $\begin{array}{c}44,49,54,59,64,69,74,79,84,89,94,99,104,109,114,119,124,129,134,139,144, \\
149,154,159,164,169,174,179,184,189\end{array}$ \\
\hline 8 & $\begin{array}{l}3,9,15,26,35,41,51,56,61,70,80,110,120,130,140,165,170,180,185,190,199,204 \\
209,214,219\end{array}$ \\
\hline 9 & $\begin{array}{l}\text { 76,81,86,91,96,101,106,111,116,121,126,131,136,141,146,151,156,161,166, } \\
171,176,181,186,191,196,201,206,211,216,221\end{array}$ \\
\hline 10 & $\begin{array}{l}17,22,27,32,37,42,47,52,57,62,67,72,77,82,87,92,97,102,107,112,117,122,127 \\
132,137,142,147,152,157,162,167,172,177,182,187,192,197,202,207,212\end{array}$ \\
\hline 11 & $\begin{array}{l}19,24,29,34,39,44,49,54,59,64,69,74,79,84,89,94,99,104,109,114,119,124,129 \\
134,139,144,149,154,159,164,169,174,179,184,189,194,199,204,209,214\end{array}$ \\
\hline 12 & $\begin{array}{l}1,6,11,16,21,26,31,36,41,46,51,56,61,66,71,76,81,86,91,96,101,106,111,116, \\
121,126,131,136,141,146,151,156,161,166,171,176,181,186,191,196,201,206 \\
211,216,221\end{array}$ \\
\hline 13 & $2,10,19,27,36,41,46,52,60,65,70,77,95,112,120,127,136,147,161,167$ \\
\hline 14 & $\begin{array}{l}\text { 43,48,53,58,63,68,73,78,83,88,93,98,103,108,113118,123,128,133,138,143, } \\
148,153,158,163,168,173,178,183,188\end{array}$ \\
\hline 15 & $\begin{array}{l}8,17,23,31,38,47,55,62,67,72,85,92,97,105,117,135,142,157,162,172,177,182 \\
, 187,192,197\end{array}$ \\
\hline 16 & $5,14,19,29,45,50,60,90,100,145,150,175,194,203,208$ \\
\hline 17 & 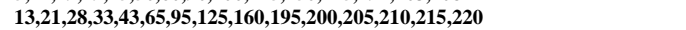 \\
\hline 18 & 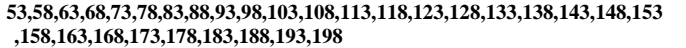 \\
\hline 19 & $1,10,16,22,27,34,40,46,52,57,75,82,87,102,107,112,122,127,137,167$ \\
\hline 20 & 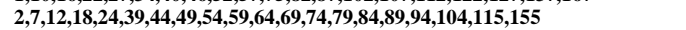 \\
\hline 21 & $\begin{array}{l}6,15,20,25,30,36,41,48,55,61,66,71,80,85,105,110,120,130,135,140,165,170 \\
180,185,190\end{array}$ \\
\hline
\end{tabular}

Table V: Channel assignment for benchmark problem 6

\begin{tabular}{|c|c|}
\hline Cells & Frequency \\
\hline 1 & $10,17,31,51,61,68,89,271$ \\
\hline 2 & $\begin{array}{l}\text { 6,19,46,59,76,96,103,118,131,139,150,164,180,187,194,201,208,220,227, } \\
234,241,248,255,262,269\end{array}$ \\
\hline 3 & $11,26,54,62,109,122,129,167$ \\
\hline 4 & $7,16,42,68,142,236,272,326$ \\
\hline 5 & $34,83,137,170,194,220,248,286$ \\
\hline 6 & $1,11,22,29,45,60,69,76,88,97,161247,278,321,338$ \\
\hline 7 & $20,27,39,49,82,104,112,119,128,135,142,159,166,181,191,202,209,250$ \\
\hline 8 & $\begin{array}{l}3,12,25,33,41,53,66,73,80,87,94,101,108,115,125,146,153,174 \\
216,223,230,237,244,258,265,279,300,328,335,342,349,356,363,370\end{array}$ \\
\hline 9 & 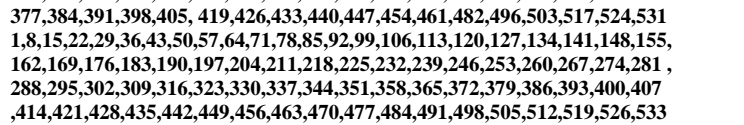 \\
\hline 10 & $\begin{array}{l}\mathbf{5 , 2 4 , 3 1 , 3 8 , 4 5 , 7 4 , 8 1 , 8 8 , 9 5 , 1 0 2 , 1 1 7 , 1 2 4 , 1 3 8 , 1 4 5 , 1 5 7 , 1 7 1 , 1 7 8 , 1 8 5 , 1 9 2 , 1 9 9 , 2 0 6} \text {, } \\
213,222,229,243,257,264,276\end{array}$ \\
\hline 11 & $13,20,28,40,49,56,65,72,79,115,153,227,310$ \\
\hline 12 & $1,30,43,63,86,111,146,158,186,200,209,224,250,270,282$ \\
\hline 13 & $\begin{array}{l}7,16,32,42,52,64,73,83,95,106,118,130,145,156,170,178,186,193,201,210,225,241, \\
254,263,273,287,310,335,355,367,375\end{array}$ \\
\hline 14 & $5,13,24,35,47,54,67,86,93,100,121,138,147,195,205$ \\
\hline 15 & $\begin{array}{l}9,18,30,37,44,56,63,70,77,84,91,98,110,117,132,140,149,163,177,184,198,212,219 \\
, 226,233,240,252,261,268,275,282,289,296,307,315,322,\end{array}$ \\
\hline 16 & $\begin{array}{l}123,130,137,144,151,158,165,172,179,186,193,200,207,214,221,228,235,242,249 \\
256,263,270,277,284,291,298,305,312,319,326,333,340,347,354,361,368,375,382,389 \\
396,403,410,417,424,431,438,445,452,459,466,473,480,487,494,501,508,515\end{array}$ \\
\hline 17 & $\begin{array}{l}10,17,52,60,69,83,90,97,104,111,160,181,188,195,202,209,251,286,293,314,321,412, \\
468,475,489,510,521,528\end{array}$ \\
\hline 18 & $2,35,47,58,67,76,119,341$ \\
\hline 19 & $2,11,26,34,42,51,58,68,75,88$ \\
\hline 20 & $4,13,23,32,39,46,54,65,72,93,100,139,329$ \\
\hline 21 & $6,19,27,37,44,63,80,87$ \\
\hline
\end{tabular}


C2

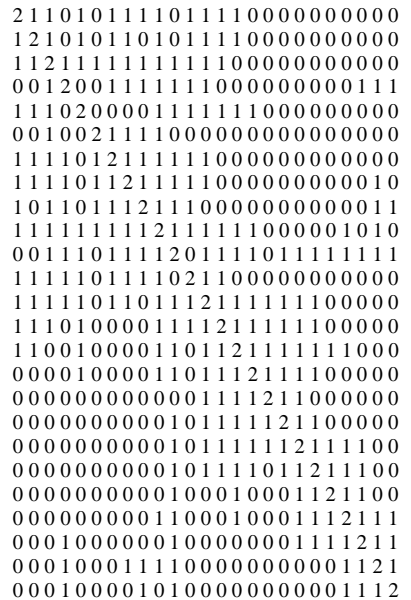

C4

711001111000011100000 171100111100001110000 117110011110000111000 011710001111000011000 001170000111000001000 100007110000111000000 110001711000111100100 111001171100011110110 11110011711000111111 01111001171100011101 001110001171000011001 000110000117000001000 000001100000711000000 100001110000171100100 110001111000117110110 11100011110001171111 0.110001111000117111 01100011100011711 00000011100001111071 0000011100011117 000000001110000111117
D2

C3

511001111000011100000 151100111100001110000 115110011110000111000 115110011110000111000 011150000111000001000 10000511000011100000 100005110000111000000 110001511000111100100 111001151100011110110 1111001151100011111 01111001151100011101 00111000115100001100 000110000115000001000 000001100000511000000 100001110000151100100 110001111000115110110 11100011110001151111 01110001111000115111 00111000111100011501 00000011100001111051 00000001110000111115 000000001110000111115

D3

721001221000011100000 272100122100001110000 127210012210000111000 012720001221000011000 001270000122000001000 100007210000221000000 210002721000122100100 221001272100012210110 12210012721000122111 01221001272100012201 001220001272000012001 000120000127000001000 000002100000721000000 100002210000272100100 110001221000127210210 110001221000127210210 11100012210001272122 011100012210001272122 00000011100001221072 0000011100001221072 00000000011100000122127

Fig. 11: Interference matrix (C) and demand vector (D) for the tested instances

\section{REFERENCES}

[1] Audhya, G. K., Sinha, K., Ghosh, S. C. and Sinha, B. P. (2011), A survey on the channel assignment problem in wireless networks. Wireless Communications and Mobile Computing, vol. 11 pp. 583 609

[2] K. I. Aardal, C.P.M. van Hoesel, A.M.C.A. Koster, C. Mannino, and A. Sassano, "Models and solution techniques for frequency assignment problems," Annals of Operations Research, 2007, vol.153, pp.79-129.

[3] Katzela I., and Nagshineh M., Channel assignment schemes for cellular mobile telecommunication systems, a comprehensive survey, IEEE Personal Communications, 1996, pp.10-31.

[4] Hale WK Frequency assignment: theory and applications Proc IEEE, 1996 vol. 68(12) pp.1497-1514.

[5] R. Montemanni, J.N.J. Moon and D. H. Smith, "An improved tabu search algorithm for the fixed spectrum frequency assignment problem", IEEE Transactions on Vehicular Technology, vol. 52(4), pp.891-901

[6] Yuanyuan Zhang; Ming Chen; , "A Metaheuristic approach for the Frequency Assignment Problem," 6th International Conference on Wireless Communications Networking and Mobile Computing (WiCOM), Sept. 2010, vol., no., pp.1-5, pp.23-25

[7] Cheng R-H., Yu C. W., Wu T-K, "A Novel Approach to the Fixed Channel Assignment Problem". Journal of Information Science and Engineering 2005, 21, pp.39-58.
[8] A.Gamst, "Homogeneous distribution of frequencies in a regular hexagonal cell system," IEEE Trans. Veh. Technol, Aug. 1982, vol. VT-31, pp. 132-144

[9] Ngo C. Y. and Li, V. O. K. "Fixed channel assignment in cellular radio networks using a modified genetic algorithm", IEEE Transactions on Vehicular Technology, 1998, 47, pp.163-171.

[10] Kim J.-S., Park S. H, Dowd P. W., and Nasrabadi N. M. "Cellular radio channel assignment using a modified hopfield network", IEEE Transactions on Vehicular Technology, 1997, 46, 4, pp.957-967.

[11] L.M. San Jose'-Revuelta "A new adaptive genetic algorithm for fixed channel assignment" Information Sciences, 2007 , vol. 177, pp. 2655-2678

[12] Eisenblätter, A., Koster, A. Web site in which the Philadelphia instances for the FAP problem are explained http://fap.zib.de/problems/Philadelphia, May 2007

[13] Moscato, P. (1989) "On Evolution, Search, ptimization, Genetic Algorithms and MartialArts: Towards Memetic Algorithms" Caltech Concurrent Computation Program, C3P Report 826.

[14] N. Krasnogor and J. Smith, "A Tutorial for Competent Memetic Algorithms: Model, Taxonomy and Design Issues," IEEE Transactions on Evolutionary Computation, 2005, vol. 9, no. 5, pp. $474-488$.

[15] Maninder Singh Kamboj, Jyotsna Sengupta, "Comparative Analysis of Simulated Annealing and Tabu Search Channel Allocation Algorithms", International Journal of Computer Theory and Engineering (IJCTE), 2009, Vol. 1, No. 5, pp. 588-591,

[16] Sivarajan, K.N., McEliece, R.J. and Ketchun, J.W. 'Channel assignment in cellular radio', Proceedings of the 39th IEEE Vehicular Technology Conference, May 1989, pp.846-850. 